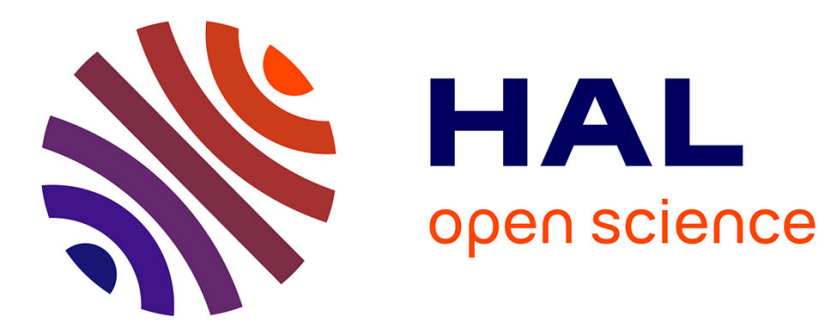

\title{
Input-to-state stabilization of nonlinear systems using event-triggered output feedback controllers
}

Mahmoud Abdelrahim, Romain Postoyan, Jamal Daafouz, Dragan Nesic

\section{To cite this version:}

Mahmoud Abdelrahim, Romain Postoyan, Jamal Daafouz, Dragan Nesic. Input-to-state stabilization of nonlinear systems using event-triggered output feedback controllers. 14th European Control Conference, ECC'15, Jul 2015, Linz, Austria. hal-01136404

\section{HAL Id: hal-01136404 https://hal.science/hal-01136404}

Submitted on 27 Mar 2015

HAL is a multi-disciplinary open access archive for the deposit and dissemination of scientific research documents, whether they are published or not. The documents may come from teaching and research institutions in France or abroad, or from public or private research centers.
L'archive ouverte pluridisciplinaire HAL, est destinée au dépôt et à la diffusion de documents scientifiques de niveau recherche, publiés ou non, émanant des établissements d'enseignement et de recherche français ou étrangers, des laboratoires publics ou privés. 


\title{
Input-to-state stabilization of nonlinear systems using event-triggered output feedback controllers
}

\author{
Mahmoud Abdelrahim, Romain Postoyan, Jamal Daafouz and Dragan Nešić
}

\begin{abstract}
We synthesize robust output-based event-triggered controllers for a class of nonlinear systems subject to external disturbances and noises in both the plant measurement and the control input. We follow an emulation approach to this purpose: we first assume that we know a robust feedback law in continuous time, we then take into account the sampling and we explain how to construct the triggering condition to preserve stability. The triggering strategy enforces a minimum time between two consecutive transmissions by combining ideas from event-triggered control and periodic sampled-data control. The closed-loop system is shown to satisfy an input-to-state stability (ISS) property with respect to the external disturbances, the noises as well as their time-derivatives. The analysis reveals a tradeoff between the enforced minimum inter-transmission time and the magnitude of the ISS gains. The results are also new in the particular case where the triggering condition is only time-dependent as in traditional sampled-data control.
\end{abstract}

\section{INTRODUCTION}

Event-triggered control is a sampling paradigm in which the sequence of transmission instants is determined based on the violation of a state-dependent criterion. In that way, a substantial reduction of the amount of transmissions may be achieved compared to conventional time-triggered setups as the loop is closed only when it is necessary in view of the control objective, see e.g., [1]-[5] and the references therein. Event-triggered implementations are particularly interesting for networked control systems and embedded systems due to their limited computation and communication resources.

Any event-triggered controller should ensure the existence of a minimum amount of time between two consecutive transmissions. This property is essential for several reasons. First, it rules out Zeno phenomenon, second it makes the hybrid controller realizable as the hardware cannot generate transmissions which are arbitrarily close in time. The problem becomes particularly non-trivial when the plant is affected by unknown exogenous inputs. It is shown in [6], for instance, that the popular technique of [3] is non-robust to external disturbances as it may generate Zeno phenomenon. Similar conclusions are presented in [7] when unmodeled

M. Abdelrahim, R. Postoyan and J. Daafouz are with the Université de Lorraine, CRAN, UMR 7039 and the CNRS, CRAN, UMR 7039, France \{othmanab1, romain.postoyan, jamal.daafouz\}@ univ-lorraine.fr. J. Daafouz is also with the Institut Universitaire de France (IUF). This work was supported by the European Community's Seventh Framework Programme (FP7/2007-2013) under grant agreement No. 257462: HYCON2 Network of Excellence "Highly-Complex and Networked Control Systems" and by the ANR under the grant COMPACS (ANR-13-BS03-0004-02).

D. Nešić is with the Department of Electrical and Electronic Engineering, the University of Melbourne, Parkville, VIC 3010, Australia dnesic@unimelb. edu . au. His work is supported by the Australian Research Council under the Discovery Projects. uncertainties are present. Several works have therefore proposed event-triggered control solutions which are robust to exogenous inputs, see e.g., [6], [8]-[14]. Most of these references assume that the full state of the plant can be measured, see [10], [11], [13], [14]. Our objective is to go beyond this assumption and to construct event-triggered output-based controllers to stabilize a class of nonlinear systems subject to external disturbances and corrupted measurements of the plant output and the control input. It is more challenging in this case to guarantee the existence of a minimum time between two consecutive transmissions when only an output of the plant is available, even for the disturbance free case as shown in [12]. Related results of the literature mainly focus on linear time-invariant (LTI) systems, see [6], [8], [9], [12]. Compared to the latter results, we address nonlinear systems, we investigate a different setup and we propose a different type of triggering conditions. Furthermore, we also take into account the possible corruption of the control input by an external signal which is not the case in the aforementioned references. Considering this type of exogenous signals is useful in practice as the control input may be subject to computation error or quantization error.

The solution we propose is inspired by our recent work in [15] which did not consider systems with exogenous inputs. We follow an emulation approach, as we start from a robust feedback law which is designed in continuous time, we then take sampling into account and we design the triggering condition to preserve stability. The triggering condition consists in waiting a fixed amount of time $T$ after each transmission instant and then to evaluate a criterion which only involves the noisy output of the plant (and potentially the noisy control input). We show that the system satisfies an ISS property with respect to the external disturbances, as well as the different noises and their time-derivative. The fact that the guaranteed stability property involves the time-derivative of the noises is common in the sampleddata control literature, see [16] for instance. Furthermore, the analysis reveals a tradeoff between the ISS gains and the guaranteed minimum time $T$ between transmissions. On the other hand, the triggering condition we propose can easily be made time-dependent only. In that way, we obtain a periodic sampled-data controller which generalizes the results in [17] to systems with exogenous inputs.

While the same type of transmission rule is proposed in [15], the fact that the overall system is affected by exogenous inputs leads to non-trivial challenges. Indeed, we first need to carefully select the coordinates of the hybrid dynamical model, in particular the sampling-induced errors. 
This point is essential for the construction of the eventtriggering condition and the stability analysis. Second, the thorough analysis in [15] has to be revisited because global asymptotic stability of nonlinear systems does not imply ISS with respect to bounded inputs, even for continuoustime systems, see [18], [19] for counter examples. Third, the presence of exogenous inputs may lead to the Zeno phenomenon as shown in [6]. Finally, we have to adapt the lower bound $T$ on the inter-transmission times in order to cope with the exogenous inputs (we cannot take the same value as in [15]).

The remainder of the paper is organised as follows. Preliminaries are given in Section II. The problem is formally stated in Section III. In Section IV, we give the main results and an illustrative example is provided in Section V. Conclusions are provided in Section VI. The proofs are given in the Appendix.

\section{Preliminaries}

Let $\mathbb{R}:=(-\infty, \infty), \mathbb{R}_{>0}:=[0, \infty)$ and $\mathbb{Z}_{\geq 0}:=$ $\{0,1,2, .$.$\} . The Euclidean norm is denoted |.|. A continuous$ function $\gamma: \mathbb{R}_{>0} \rightarrow \mathbb{R}_{>0}$ is of class $\mathcal{K}$ if it is zero at zero, strictly increasing, and it is of class $\mathcal{K}_{\infty}$ if in addition $\gamma(s) \rightarrow$ $\infty$ as $s \rightarrow \infty$. A continuous function $\gamma: \mathbb{R}_{>0} \times \mathbb{R}_{>0} \rightarrow \mathbb{R}_{>0}$ is of class $\mathcal{K} \mathcal{L}$ if for each $t \in \mathbb{R}_{\geq 0}, \gamma(., t)$ is of class $\mathcal{K}$, and, for each $s \in \mathbb{R}_{\geq 0}, \gamma(s,$.$) is decreasing to zero. We denote$ the minimum and maximum eigenvalues of the square, real, symmetric matrix $A$ as $\lambda_{\min }(A)$ and $\lambda_{\max }(A)$, respectively. We write $(x, y)$ to represent the vector $\left[x^{T}, y^{T}\right]^{T}$ for $x \in \mathbb{R}^{n}$ and $y \in \mathbb{R}^{m}$. We will consider locally Lipschitz Lyapunov functions (that are not necessarily differentiable everywhere), therefore we will use the generalized directional derivative of Clarke which is defined as follows. For a locally Lipschitz function $V: \mathbb{R}^{n} \rightarrow \mathbb{R}_{>0}$ and a vector $v \in \mathbb{R}^{n}, V^{\circ}(x ; v):=$ $\lim \sup _{h \rightarrow 0^{+}, y \rightarrow x}(V(y+h v)-V(y)) / h$. For a continuously differentiable function $V, V^{\circ}(x ; v)$ reduces to the standard directional derivative $\langle\nabla V(x), v\rangle$, where $\nabla V(x)$ is the (classical) gradient. We will invoke the following result, see Lemma II.1 in [20].

Lemma 1 (Lemma II.1 [20]). Consider two functions $U_{1}$ : $\mathbb{R}^{n} \rightarrow \mathbb{R}$ and $U_{2}: \mathbb{R}^{n} \rightarrow \mathbb{R}$ that have well-defined Clarke derivatives for all $x \in \mathbb{R}^{n}$ and $v \in \mathbb{R}^{n}$. Introduce three sets $A:=\left\{x: U_{1}(x)>U_{2}(x)\right\}, B:=\left\{x: U_{1}(x)<U_{2}(x)\right\}$, $\Gamma:=\left\{x: U_{1}(x)=U_{2}(x)\right\}$. Then, for any $v \in \mathbb{R}^{n}$, the function $U(x):=\max \left\{U_{1}(x), U_{2}(x)\right\}$ satisfies $U^{\circ}(x ; v)=$ $U_{1}^{\circ}(x ; v)$ for all $x \in A, U^{\circ}(x ; v)=U_{2}^{\circ}(x ; v)$ for all $x \in B$ and $U^{\circ}(x ; v) \leq \max \left\{U_{1}^{\circ}(x ; v), U_{2}^{\circ}(x ; v)\right\}$ for all $x \in \Gamma$.

In this paper, we consider hybrid systems of the following form using the formalism of [21], [22]

$$
\dot{x}=F(x, u) \quad x \in C, \quad x^{+}=G(x, u) \quad x \in D,
$$

where $x \in \mathbb{R}^{n_{x}}$ is the state, $u \in \mathbb{R}^{n_{u}}$ is the input, $C$ is the flow set, $F$ is the flow map, $D$ is the jump set and $G$ is the jump map. The vector fields $F$ and $G$ are assumed to be continuous and the sets $C$ and $D$ are closed. The solutions to system (1) are defined on so-called hybrid time domains. A set $E \subset \mathbb{R}_{\geq 0} \times \mathbb{Z}_{\geq 0}$ is called a compact hybrid time domain if $E=\bigcup_{j=0}^{J-1}\left(\left[t_{j}, t_{j+1}\right], j\right)$ for some finite sequence of times $0=t_{0} \leq t_{1} \leq \ldots \leq t_{J}$ and it is a hybrid time domain if for all $(T, J) \in E, E \cap([0, T] \times\{0,1, \ldots, J\})$ is a compact hybrid time domain.

A hybrid signal is a function defined on a hybrid time domain. A hybrid signal $u: \operatorname{dom} u \rightarrow \mathbb{R}^{n_{u}}$ is called a hybrid input (or disturbance) if $u(., j)$ is measurable and locally essentially bounded for each $j$. A hybrid signal $x: \operatorname{dom} x \rightarrow$ $\mathbb{R}^{n_{x}}$ is called a hybrid arc if $x(., j)$ is locally absolutely continuous for each $j$. A hybrid arc $x: \operatorname{dom} x \rightarrow \mathbb{R}^{n_{x}}$ is a solution to system (1) with hybrid input $u: \operatorname{dom} u \rightarrow \mathbb{R}^{n_{u}}$ if $\operatorname{dom} x=\operatorname{dom} u, x(0,0) \in C \cup D$, and: (i) for all $j \in \mathbb{Z}_{\geq 0}$, and almost all $t$ such that $(t, j) \in \operatorname{dom} x, x(t, j) \in C$ and $\dot{x}(t, j)=F(x(t, j), u(t, j))$; (ii) for all $(t, j) \in \operatorname{dom} x$ such that $(t, j+1) \in \operatorname{dom} x, x(t, j) \in D$ and $x(t, j+1)=$ $G(x(t, j), u(t, j))$. The following definition of $\mathcal{L}_{\infty}$ norm is considered in [22], [23] for hybrid signals.

Definition 1. Given a hybrid signal $r$, its $\mathcal{L}_{\infty}$ norm is given by

$$
\|r\|_{(t, j)}:=\max \left\{\begin{array}{l}
\sup _{\left(t^{\prime}, j^{\prime}\right) \in \operatorname{dom} r \backslash \Gamma(r), t^{\prime}+j^{\prime} \leq t+j}\left|r\left(t^{\prime}, j^{\prime}\right)\right|, \\
\left.\sup _{\left(t^{\prime}, j^{\prime}\right) \in \Gamma(r), t^{\prime}+j^{\prime} \leq t+j}\left|r\left(t^{\prime}, j^{\prime}\right)\right|\right\},
\end{array}\right.
$$

where $\Gamma(r):=\{(t, j) \in \operatorname{dom} r:(t, j+1) \in \operatorname{dom} r\}$. Furthermore, a hybrid input $u$ is said to belong to $\mathcal{L}_{\infty}$ when $\|u\|_{(t, j)} \leq c$ for any $(t, j) \in \operatorname{dom} u$, for some $c \geq 0$.

\section{Problem Statement}

Consider the nonlinear plant model

$$
\dot{x}_{p}=f_{p}\left(x_{p}, u, w\right), \quad y=g_{p}\left(x_{p}, d_{y}\right),
$$

where $x_{p} \in \mathbb{R}^{n_{p}}$ is the plant state, $u \in \mathbb{R}^{n_{u}}$ is the control input, $w \in \mathbb{R}^{n_{w}}$ is a vector of unknown exogenous disturbances, $y \in \mathbb{R}^{n_{y}}$ is the available output of the plant which is affected by the vector of noises $d_{y} \in \mathbb{R}^{n_{d_{y}}}$. We consider the following dynamic controller

$$
\dot{x}_{c}=f_{c}\left(x_{c}, y\right), \quad u=g_{c}\left(x_{c}, y, d_{u}\right),
$$

where $x_{c} \in \mathbb{R}^{n_{c}}$ is the controller state and $d_{u} \in \mathbb{R}^{n_{d_{u}}}$ is a measurement noise affecting the control input. The presence of $d_{u}$ may be due to quantization error or computational glitch, to give a few examples. We emphasize that the $x_{c}$-system is not necessarily an observer and that (4) captures static feedbacks laws as a particular case by setting $u=g_{c}\left(y, d_{u}\right)$. We assume that the signals corresponding to $w, d_{y}, d_{u}$ are unknown, bounded (according to the $\mathcal{L}_{\infty^{-}}$ norm), differentiable for $d_{y}$ and $d_{u}$ with time-derivatives in $\mathcal{L}_{\infty}$. Note that we do not need to know any bound on the norm of these signals to apply the presented results. The functions $f_{p}, f_{c}$ are assumed to be continuous and the functions $g_{p}, g_{c}$ are assumed to be continuously differentiable.

We consider the scenario where controller (4) communicates with the plant via a digital channel. Hence, the plant 
output $y$ and the control input $u$ are sent only at transmission instants $t_{i}, i \in \mathbb{Z}_{\geq 0}$. We are interested in an event-triggered implementation in the sense that the sequence of transmission instants is determined by a criterion based on the output measurement $y$ and the control input $u$, see Figure 1 . Note that the event-triggering mechanism only depends on the noisy variables $y$ and $u$.

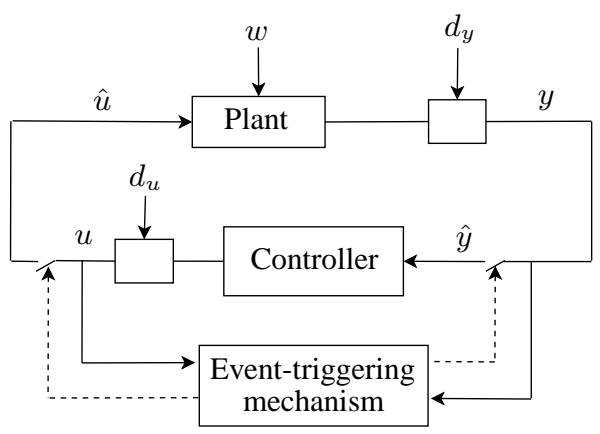

Fig. 1. Event-triggered control schematic.

The setup we envision allows the triggering condition to depend on both $y$ and $u$. While this may be hard to achieve in practice, we focus on this general scenario because it allows us to cover the particular cases where the triggering condition only depends on $y$ or $u$ as particular cases. This will be the case for instance when the controller is static or when only $y$ or $u$ is sampled, to give a few examples.

At each transmission instant, $y$ is sent to the controller which computes a new control input that is instantaneously transmitted to the plant. In that way, we obtain

$$
\begin{aligned}
\dot{x}_{p} & =f_{p}\left(x_{p}, \hat{u}, w\right) \\
\dot{x}_{c} & =f_{c}\left(x_{c}, \hat{y}\right) \\
u & =g_{c}\left(x_{c}, \hat{y}, d_{u}\right) \\
y & =g_{p}\left(x_{p}, d_{y}\right) \\
\dot{\hat{y}} & =0 \\
\dot{\hat{u}} & =0 \\
\hat{y}\left(t_{i}^{+}\right) & =y\left(t_{i}\right) \\
\hat{u}\left(t_{i}^{+}\right) & =u\left(t_{i}\right),
\end{aligned}
$$

where $\hat{y}$ and $\hat{u}$ respectively denote the last transmitted values of $y$ and $u$. We assume that zero-order-hold devices are used to generate the sampled values $\hat{y}$ and $\hat{u}$, which leads to $\dot{\hat{y}}=0$ and $\dot{\hat{u}}=0$.

Because we only have access to noisy signals, we need to define the sampling-induced error using noisy variables. Hence, we consider $e:=\left(e_{y}, e_{u}\right) \in \mathbb{R}^{n_{e}}$, where

$$
\begin{aligned}
e_{y} & :=\hat{y}-y \\
e_{u} & :=\hat{u}-u
\end{aligned}
$$

which is reset to 0 at each transmission instant. Note that $e$ is available to the event-triggering mechanism.

We model the event-triggered controlled system using the hybrid formalism of [21] like in [15], [12], [24], [4], for which a jump corresponds to a transmission. In that way, we obtain

$$
\begin{aligned}
\left(\begin{array}{c}
\dot{x} \\
\dot{e} \\
\dot{\tau}
\end{array}\right) & =\left(\begin{array}{c}
f(x, e, w, d) \\
g(x, e, w, d, v) \\
1
\end{array}\right) \quad(x, e, \tau) \in C \\
\left(\begin{array}{c}
x^{+} \\
e^{+} \\
\tau^{+}
\end{array}\right) & =\left(\begin{array}{c}
x \\
0 \\
0
\end{array}\right) \quad(x, e, \tau) \in D,
\end{aligned}
$$

where $\tau$ is an additional clock variable introduced to describe the time elapsed since the last transmission, $x:=\left(x_{p}, x_{c}\right) \in$ $\mathbb{R}^{n_{x}}, d:=\left(d_{y}, d_{u}\right) \in \mathbb{R}^{n_{d}}, v:=\left(v_{y}, v_{u}\right) \in \mathbb{R}^{n_{d}}$ and $v_{y}, v_{u}$ respectively denote the time-derivative of the noises corresponding to $d_{y}$ and $d_{u}$. The functions $f, g$ in (7) are given by (their arguments are omitted below)

$$
\begin{aligned}
& f=\left(\begin{array}{c}
f_{p}\left(x_{p}, g_{c}\left(x_{c}, y+e_{y}, d_{u}\right)+e_{u}, w\right) \\
f_{c}\left(x_{c}, y+e_{y}\right)
\end{array}\right) \\
& g=\left(\begin{array}{c}
-\frac{\partial}{\partial x_{p}} g_{p}\left(x_{p}, d_{y}\right) f_{p}\left(x_{p}, g_{c}\left(x_{c}, y+e_{y}, d_{u}\right)+e_{u}, w\right) \\
-\frac{\partial}{\partial d_{y}} g_{p}\left(x_{p}, d_{y}\right) v_{y} \\
-\frac{\partial}{\partial x_{c}} g_{c}\left(x_{c}, y+e_{y}, d_{u}\right) f_{c}\left(x_{c}, y+e_{y}\right) \\
-\frac{\partial}{\partial d_{u}} g_{c}\left(x_{c}, y+e_{y}, d_{u}\right) v_{u}
\end{array}\right) .
\end{aligned}
$$

The flow and jump sets of (7) are defined according to the triggering condition we will synthesize. As long as the triggering condition is not violated, the system flows on $C$ and a jump occurs when the state enters in $D$. When $(x, e, \tau) \in C \cap D$, the solution may flow only if flowing keeps $(x, e, \tau)$ in $C$, otherwise the system experiences a jump. The sets $C$ and $D$ will be closed.

The main objective of this paper is to design the flow and the jump sets of system (7), i.e. the triggering condition, to ensure an ISS stability property for system (7) as well as to ensure the existence of a strictly positive lower bound on the inter-transmission times.

\section{MAIN RESUlts}

We first present the conditions we assume on system (7), then we present the event-triggering law and we state the main result. Finally, we explain how to apply our results in the periodic sampled-data control.

\section{A. Assumptions}

We make the following assumption on system (7), which is a generalization of Assumption 1 in [17].

Assumption 1. There exist a locally Lipschitz positive definite function $V: \mathbb{R}^{n_{x}} \rightarrow \mathbb{R}_{\geq 0}$, a locally Lipschitz positive semi-definite function $W: \mathbb{R}^{n_{e}} \rightarrow \mathbb{R}_{\geq 0}$, a continuous function $H: \mathbb{R}^{n_{x}} \rightarrow \mathbb{R}_{\geq 0}$, real numbers $L \geq 0, \gamma>0$, $\underline{\alpha}, \bar{\alpha}, \alpha, \mu, \sigma \in \mathcal{K}_{\infty}$ and $\bar{a}$ continuous, nonnegative function $\bar{\delta}: \mathbb{R}^{n_{y}} \rightarrow \mathbb{R}_{>0}$ such that the following holds.

(i) For all $x \in \mathbb{R}^{n_{x}}$

$$
\underline{\alpha}(|x|) \leq V(x) \leq \bar{\alpha}(|x|) .
$$


(ii) For almost all $x \in \mathbb{R}^{n_{x}}$ and all $(e, w, d, v) \in$ $\mathbb{R}^{n_{e}+n_{w}+2 n_{d}}$

$$
\begin{aligned}
\langle\nabla V(x), f(x, e, w, d)\rangle \leq & -\alpha(|x|)-H^{2}(x)-\delta(y) \\
& +\gamma^{2} W^{2}(e)+\mu(|(w, d, v)|) .
\end{aligned}
$$

(iii) For almost all $e \in \mathbb{R}^{n_{e}}$ and all $(x, w, d, v) \in$ $\mathbb{R}^{n_{x}+n_{w}+2 n_{d}}$

$$
\begin{aligned}
\langle\nabla W(e), g(x, e, w, d, v)\rangle \leq & L W(e)+H(x) \\
& +\sigma(|(w, d, v)|) .
\end{aligned}
$$

The function $V$ in Assumption 1 corresponds to a Lyapunov function for the closed-loop system in the absence of sampling. Property (10) implies that the system $\dot{x}=$ $f(x, e, w, d)$ is $\mathcal{L}_{2}$-gain stable from $(W, \sqrt{\mu})$ to $(H, \sqrt{\delta})$. Items (i) and (ii) of Assumption 1 also imply that the system $\dot{x}=f(x, e, w, d)$ satisfies a derivative ISS property, in the sense of [25], with respect to $w, d$ (since $\mu \in \mathcal{K}_{\infty}$ and $W$ is positive semi-definite and continuous). It has to be noted that the case where item (ii) of Assumption 1 holds with $\mu(|(w, d)|)$ instead of $\mu(|(w, d, v)|)$, is a particular case of (10). We decided to consider the case where $\mu$ also depends on $v$ for the sake of generality. The last item of Assumption 1 is an exponential growth condition on the $e$-system, similar conditions are imposed in [17]. Note that the dependence of $\sigma$ on $v$ comes from the sampling of the noisy measurements, see (6), which is typical when investigating sampled-data systems with external inputs, see for instance [16].

\section{B. Triggering condition}

Inspired by [15], we trigger transmissions whenever the condition below is violated

$$
\gamma^{2} W^{2}(e) \leq \delta(y) \text { or } \tau \in[0, T]
$$

where $\gamma, W$ and $\delta$ come from Assumption 1 and $T>0$ is selected such that $T<\mathcal{T}(\gamma, \eta, L)$, where

$$
\mathcal{T}(\gamma, \eta, L):= \begin{cases}\frac{1}{L r} \arctan (r) & (1+\eta) \gamma>L \\ \frac{1}{L} & (1+\eta) \gamma=L \\ \frac{1}{L r} \operatorname{arctanh}(r) & (1+\eta) \gamma<L\end{cases}
$$

with $r:=\sqrt{\left|\left(\frac{(1+\eta) \gamma}{L}\right)^{2}-1\right|}$. The constant $\eta>0$ is a tuning parameter which can be arbitrarily chosen by the user. Note that $\mathcal{T}(\gamma, \eta, L)$ is a decreasing function in $\eta$ and when $\eta \rightarrow$ 0 we recover the upper bound on the maximum allowable transmission interval (MATI) given by [17] and used in [15]. Hence, a small value of $\eta>0$ leads to a larger $\mathcal{T}(\gamma, \eta, L)$, however this will lead to a big ISS gain as we will see below. The constant $\mathcal{T}(\gamma, \eta, L)$ is therefore different from the one in [15], which means that we have to adapt the enforced minimum time between two successive transmissions to the presence of exogenous inputs.
In view of (12), the flow and jump sets of system (7) are

$$
\begin{aligned}
& C=\left\{(x, e, \tau): \gamma^{2} W^{2}(e) \leq \delta(y) \text { or } \tau \in[0, T]\right\} \\
& D=\left\{(x, e, \tau): \gamma^{2} W^{2}(e) \geq \delta(y) \text { and } \tau \geq T\right\} .
\end{aligned}
$$

\section{Stability result}

We are ready to state the main result. Its proof is provided in the Appendix.

Theorem 1. Suppose that Assumption 1 holds and consider system (7) with the flow and jump sets defined in (14), where the constant $T$ is such that $T \in(0, \mathcal{T}(\gamma, \eta, L))$. There exist $\beta \in \mathcal{K} \mathcal{L}$ and $\xi \in \mathcal{K}_{\infty}$ such that any solution $\phi=$ $\left(\phi_{x}, \phi_{e}, \phi_{\tau}\right)$ with $w, d, v \in \mathcal{L}_{\infty}$ and $\operatorname{dom}(w, d, v)=\operatorname{dom} \phi$ satisfies, for all $(t, j) \in \operatorname{dom} \phi$,

$$
\begin{aligned}
& \left|\phi_{x}(t, j)\right| \leq \\
& \max \left\{\beta\left(\left|\left(\phi_{x}(0,0), \phi_{e}(0,0)\right)\right|, t+j\right), \xi\left(||(w, d, v) \|_{(t, j)}\right)\right\},
\end{aligned}
$$

where $\xi(s):=\underline{\alpha}^{-1}\left(\rho^{-1}\left(\frac{1}{\varepsilon}\left(\mu(s)+\frac{1}{\eta} \sigma^{2}(s)\right)\right)\right), \rho(s):=$ $\min \left\{\rho_{1}\left(\frac{s}{2}\right), \eta \gamma \theta \frac{s}{2}\right\}$ for $s \geq 0, \rho_{1} \in \mathcal{K}_{\infty}, \eta>0$ and $\varepsilon, \theta \in(0,1)$. Furthermore, the inter-transmission times are lower bounded by the constant $T$.

Theorem 1 shows that system (7), (14) satisfies a derivative ISS property, see [25], with respect to the external disturbance $w$ and the measurement noise $d$. It is interesting to note that even when $\mu$ in (10) is independent of $v$ (the timederivative of the noises), the ISS gain $\xi$ in (15) does depend on $v$ because of (11). This type of result is common in the sampled-data control literature, see [16]. The constants $\theta$ and $\varepsilon$ can be arbitrarily chosen in $(0,1)$ as shown in the proof. We notice, in view of (13), (15), that $\eta$ provides a tradeoff between the guaranteed minimum time between transmissions and the magnitude of the ISS gain $\xi$. The value of $\mathcal{T}(\gamma, \eta, L)$ can be increased by taking $\eta$ small, however the ISS (nonlinear) gain $\xi$ in (15) will increase, and vice versa. When $\eta \rightarrow \infty$ and $\varepsilon \rightarrow 1$ (which implies that $T \rightarrow 0$ ), we recover the ISS property ensured in the absence of sampling.

\section{Time-triggered implementation}

The results of this section covers periodic sampled-data control as a particular case by removing the output dependent condition $\gamma^{2} W^{2}(e) \leq \delta(y)$ in (12). In that way, the flow and the jump sets of system (7) are

$$
\begin{aligned}
& C=\{(x, e, \tau): \tau \in[0, T]\} \\
& D=\{(x, e, \tau): \tau=T\},
\end{aligned}
$$

and $T$ is selected strictly smaller than $\mathcal{T}(\gamma, \eta, L)$ in (13) as before. We can then state the following result which generalizes [17] to systems affected by exogenous inputs. Its proof follows the same lines as the proof of Theorem 1, it is therefore omitted.

Corollary 1. Suppose that Assumption 1 holds and consider system (7), (16). Then, the conclusions of Theorem 1 hold. 


\section{ILLUSTRATIVE EXAMPLE}

We consider the controlled Lorenz model of fluid convection in [26] affected by external disturbances and measurement noises

$$
\begin{aligned}
\dot{x}_{1} & =-a x_{1}+a x_{2}+w_{1} \\
\dot{x}_{2} & =b x_{1}-x_{2}-x_{1} x_{3}+u+w_{2} \\
\dot{x}_{3} & =x_{1} x_{2}-c x_{3}+w_{3} \\
y & =x_{1}+d_{y},
\end{aligned}
$$

where $x_{1}$ is proportional to the intensity of the convective motion, $x_{2}$ is proportional to the temperature difference between the ascending and descending currents, $x_{3}$ is proportional to the distortion of the vertical temperature profile from being linear, $u$ corresponds to the tilt angle of a closedloop of natural convection from the vertical, $w_{1}, w_{2}, w_{3}$ are external disturbances and $d_{y}$ is the measurement noise. The parameters $a, b$, and $c$ are related to some physical constants and are positive, see [27] for more detail.

We consider the following static output feedback controller $u=-\left(\frac{p_{1}}{p_{2}} a+b\right) y$, where $p_{1}, p_{2}>0$. We assume that $u$ is affected by some additive noise $d_{u}$, hence the input to (17) is

$$
u=-\left(\frac{p_{1}}{p_{2}} a+b\right) y+d_{u} .
$$

We consider the scenario where a network is used to transmit data between the plant and the controller. We therefore take into account the sampling-induced error

$$
e=\hat{y}-y \text {. }
$$

Note that it is not necessary to consider the error in $u$ as the controller is static, as explained in Section III. Denote $x:=\left(x_{1}, x_{2}, x_{3}\right), w:=\left(w_{1}, w_{2}, w_{3}\right)$ and $d:=\left(d_{y}, d_{u}\right)$. We can model the system as (7) with (the arguments of $f, g$ are omitted below)

$$
\begin{aligned}
& f=\left(\begin{array}{c}
-a x_{1}+a x_{2}+w_{1} \\
b x_{1}-x_{2}-x_{1} x_{3}-\left(\frac{p_{1}}{p_{2}} a+b\right)\left(y_{s}+e\right)+d_{u}+w_{2} \\
x_{1} x_{2}-c x_{3}+w_{3}
\end{array}\right) \\
& g=a x_{1}-a x_{2}-w_{1}-v_{y},
\end{aligned}
$$

recall that $v_{y}$ denotes the time-derivative of the signal corresponding to $d_{y}$.

Let $W(e)=|e|$ for any $e$. For all $x \in \mathbb{R}^{3}$ and almost all $e \in \mathbb{R}^{n_{e}}, v_{y} \in \mathbb{R}^{n_{d y}}$

$$
\langle\nabla W(e), g\rangle \leq a\left|x_{1}\right|+a\left|x_{2}\right|+\left|w_{1}\right|+\left|v_{y}\right| .
$$

Hence, condition (11) holds with $L=0, H(x)=a\left|x_{1}\right|+$ $a\left|x_{2}\right|, \sigma(|(w, d, v)|)=2|(w, d, v)|$. Let $V(x)=p_{1} x_{1}^{2}+$ $p_{2} x_{2}^{2}+p_{2} x_{3}^{2}$, where $p_{1}, p_{2}>0$. Item (i) in Assumption 1 holds with $\underline{\alpha}(s)=\min \left\{p_{1}, p_{2}\right\} s^{2}$ and $\bar{\alpha}(s)=$ $\max \left\{p_{1}, p_{2}\right\} s^{2}$ for any $s \geq 0$. By taking $p_{1}>2, p_{2}>$ $2 a, c>\frac{1}{2}$, item (ii) in Assumption 1 is verified with $\alpha(s)=\min \left\{\frac{1}{2} a\left(p_{1}-2\right),\left(p_{2}-2 a\right), p_{2}(2 c-1)\right\} s^{2}, \delta(y)=$ $\frac{1}{4} a\left(p_{1}-2\right) y^{2}, \gamma=\sqrt{4 p_{2}\left(\frac{p_{1}}{p_{2}} a+b\right)^{2}}$ and $\mu(|(w, d)|)=$ $\max \left\{p_{1}, 4 p_{2}\right\}|(w, d)|^{2}+\max \left\{4 p_{2}, 4 p_{2}\left(\frac{p_{1}}{p_{2}} a+b\right)^{2}+\frac{1}{2} a\left(p_{1}-\right.\right.$ $2)\}|(w, d)|^{2}$. Note that $\mu$ is independent on $v$ in this example.
We set the parameter values $a=10, b=28, c=8 / 3$, as in [26], and we take $p_{1}=3, p_{2}=3 a, \eta=0.05$. We obtain $\mathcal{T}(\gamma, \eta, L)=0.0047$ using (13). Hence, we select $T=0.0045$ and (12) becomes

$$
1.0092 \times 10^{5}|e|^{2} \leq 2.5 y^{2} \text { or } \tau \in[0,0.0045]
$$

We run simulations with random disturbances $w$ satisfying $\left|w_{i}(t)\right| \leq 0.1$ for any $t \geq 0, i \in\{1,2,3\}$ and the following noises signals $d_{y}(t)=d_{u}(t)=0.1 \sin (50 t)$ for any $t \geq 0$. Figure 2 shows that the state trajectory of the plant converges to a neighbourhood to the origin as expected, where the initial condition in this case is $(x(0,0), e(0,0), \tau(0,0))=$ $(-20,-20,30,0,0)$. The generated inter-transmission times are provided in Figure 3, where we can see the interaction between the time-dependent and the output-dependent conditions of the triggering rule. It can be also observed from Figure 3 that the minimum inter-transmission time corresponds to the guaranteed lower bound $T$, represented by the red line, which justifies our event-triggering mechanism. This point is also supported by Figure 4 where Zeno phenomenon occurs when we set $T=0$, i.e. without enforcing a minimum time between transmissions. Table I provides the minimum and the average inter-transmission times, respectively denoted as $\tau_{\min }$ and $\tau_{\text {avg }}$, for 100 randomly distributed initial conditions such that $|x(0,0)|,|e(0,0)| \leq 100$ and $\tau(0,0)=0$ for a simulation time of 10 seconds.

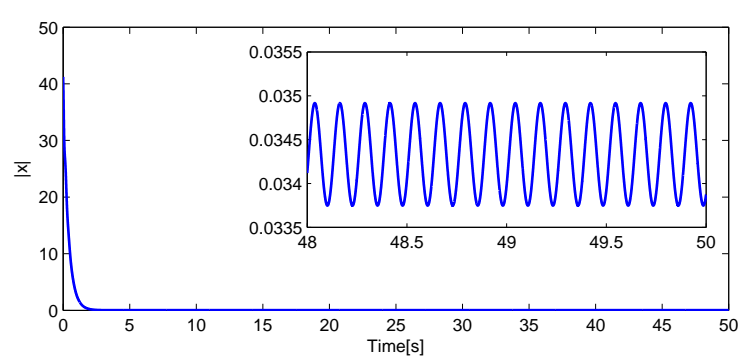

Fig. 2. State trajectory of the plant.

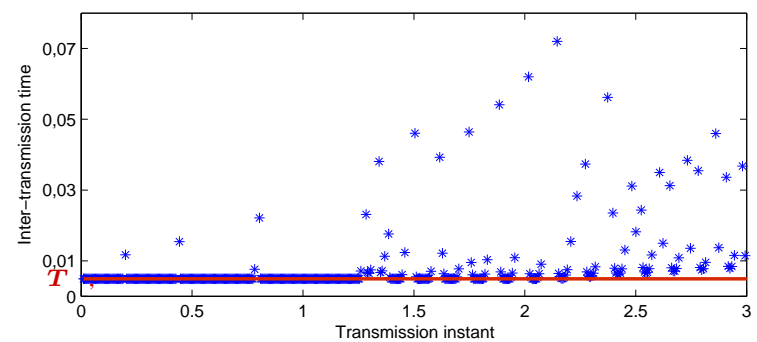

Fig. 3. Inter-transmission times for the first three units of time. 


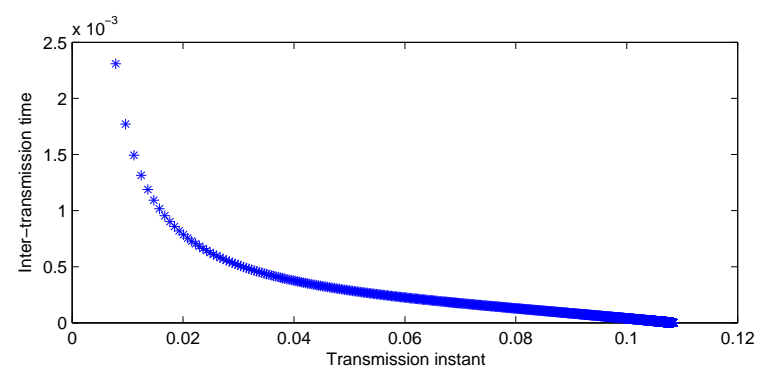

Fig. 4. Inter-transmission times with $T=0$.

\begin{tabular}{c|c|c} 
Guaranteed lower bound & $\tau_{\text {min }}$ & $\tau_{\text {avg }}$ \\
\hline 0.0045 & 0.0045 & 0.0116
\end{tabular}

TABLE I

SIMULATION RESULTS FOR 100 RANDOMLY DISTRIBUTED INITIAL CONDITIONS SUCH THAT $|x(0,0)|,|e(0,0)| \leq 100$ AND $\tau(0,0)=0$ FOR A SIMULATION TIME OF 10 SECONDS.

\section{CONClusion}

Input-to-state stabilizing event-triggered output feedback controllers have been synthesized for a class of nonlinear systems subject to external disturbances and corrupted output measurements and control inputs. The developed technique generalizes the results in [15] and enforces a strictly positive lower bound on the inter-transmission times. Future work will investigate the $\mathcal{L}_{p}$ stability of the closed-loop system and the application of the results to LTI systems in the context of co-design, like in [28].

\section{REFERENCES}

[1] K. Årzén, "A simple event-based PID controller," In Proceedings of the $14^{\text {th }}$ IFAC World Congress, Beijing, China, vol. 18, pp. 423-428, 1999.

[2] K. Åström and B. Bernhardsson, "Comparison of periodic and event based sampling for first order stochastic systems," In Proceedings of the 14th IFAC World Congress, Beijing, China, pp. 301-306, 1999.

[3] P. Tabuada, "Event-triggered real-time scheduling of stabilizing control tasks," IEEE Transactions on Automatic Control, vol. 52, no. 9, pp. 1680-1685, 2007.

[4] R. Postoyan, P. Tabuada, D. Nešić, and A. Anta, "A framework for the event-triggered stabilization of nonlinear systems," IEEE Transactions on Automatic Control, to appear.

[5] W. Heemels, K. Johansson, and P. Tabuada, "An introduction to eventtriggered and self-triggered control," In Proceedings of the IEEE Conference on Decision and Control, Hawaii, U.S.A., pp. 3270-3285, 2012.

[6] D. Borgers and W. Heemels, "Event-separation properties of eventtriggered control systems," IEEE Transactions on Automatic Control, accepted, 2014.

[7] M. Abdelrahim, R. Postoyan, and J. Daafouz, "Event-triggered control of nonlinear singularly perturbed systems based only on the slow dynamics," Automatica, accepted.

[8] J. Sijs, M. Lazar, and W. Heemels, "On integration of event-based estimation and robust MPC in a feedback loop," Hybrid Systems: Computation and control, Stockholm, Sweden, pp. 31-41, 2010.

[9] D. Lehmann and J. Lunze, "Event-based output-feedback control," In Proceedings of the 19th Mediterranean Conference on Control and Automation, Corfu, Greece, pp. 982-987, 2011.

[10] C. D. Persis, R. Sailer, and F. Wirth, "On a small-gain approach to distributed event-triggered control," In Proceedings of the 18th IFAC World Congress, Milano, Italy, pp. 2401-2406, 2011.
[11] D. Lehmann, G. Kiener, and K. Johansson, "Event-triggered pi control: Saturating actuators and anti-windup compensation," In Proceedings of the 51st Conference on Decision and Control, Hawaii, U.S.A., pp. 6566-6571, 2012.

[12] M. Donkers and W. Heemels, "Output-based event-triggered control with guaranteed $\mathcal{L}_{\infty}$-gain and improved and decentralised eventtriggering," IEEE Transactions on Automatic Control, vol. 57, no. 6, pp. 1362-1376, 2012.

[13] D. Lehmann, E. Henriksson, and K. Johansson, "Event-triggered model predictive control of discrete-time linear systems subject to disturbances," In Proceedings of the European Control Conference, Zürich, Switzerland, pp. 1156-1161, 2013.

[14] D. Shi, T. Chen, and L. Shi, "Event-triggered maximum likelihood state estimation," Automatica, vol. 50, no. 1, pp. 247-254, 2014.

[15] M. Abdelrahim, R. Postoyan, J. Daafouz, and D. Nešić, "Stabilization of nonlinear systems using event-triggered output feedback laws," In Proceedings of the 21th International Symposium on Mathematics Theory of Networks and Systems, Groningen, The Netherlands, pp. 274-281, 2014.

[16] D. Nešić and D. Laila, "A note on input-to-state stabilization for nonlinear sampled-data systems," IEEE Transactions on Automatic Control, vol. 47, no. 7, pp. 1153-1158, 2002.

[17] D. Nešić, A. Teel, and D. Carnevale, "Explicit computation of the sampling period in emulation of controllers for nonlinear sampled-data systems," IEEE Transactions on Automatic Control, vol. 54, no. 3, pp. 619-624, 2009.

[18] E. Sontag, "Input to state stability: Basic concepts and results," In P. Nistri and G. Stefani, editors, Nonlinear and Optimal Control Theory, Springer-Verlag, Berlin, pp. 163-220, 2007.

[19] R. Freeman, "Global internal stabilizability does not imply global external stabilizability for small sensor disturbances," IEEE Transactions on Automatic Control, vol. 40, pp. 2119-2122, 1995.

[20] D. Liberzon, D. Nešić, and A. Teel, "Lyapunov-based small-gain theorems for hybrid systems," IEEE Transactions on Automatic Control, vol. 59, no. 6, pp. 1395-1410, 2014.

[21] R. Goebel, R. Sanfelice, and A. Teel, Hybrid Dynamical Systems: Modeling, Stability, and Robustness. Princeton University Press, 2012.

[22] C. Cai and A. Teel, "Characterizations of input-to-state stability for hybrid systems," Systems \& Control Letters, vol. 58, no. 1, pp. 47-53, 2009.

[23] R. Sanfelice, "Results on input-to-output and input-output-to-state stability for hybrid systems and their interconnections," In Proceedings of the 49th IEEE Conference on Decision and Control, Georgia, U.S.A., pp. 2396-2401, 2010.

[24] F. Forni, S. Galeani, D. Nešić, and L. Zaccarian, "Event-triggered transmission for linear control over communication channels," Automatica, vol. 50, no. 2, pp. 490-498, 2014.

[25] A. Angeli, E. Sontag, and Y. Wang, "Input-to-state stability with respect to inputs and their derivatives," International Journal of Robust and Nonlinear Control, vol. 13, no. 11, pp. 1035-1056, 2003.

[26] C. Wan and D. Bernstein, "Nonlinear feedback control with global stabilization," Dynamics and Control, vol. 5, pp. 321-346, 1995.

[27] T. Vincent and J. Yu, "Control of a chaotic system," Dynamics and Control, vol. 1, pp. 35-52, 1991.

[28] M. Abdelrahim, R. Postoyan, J. Daafouz, and D. Nešić, "Co-design of output feedback laws and event-triggering conditions for linear systems," In Proceedings of the IEEE Conference on Decision and Control, Los Angeles, U.S.A., accepted.

[29] A. Teel and L. Praly, "On assigning the derivative of a disturbance attenuation control Lyapunov function," Mathematics of Control, Signal and Systems, vol. 13, no. 2, pp. 95-124, 2000.

[30] H. Khalil, Nonlinear Systems, 3rd ed. Prentice Hall, 2002.

\section{APPENDIX}

Proof of Theorem 1. We first introduce $\zeta: \mathbb{R}_{>0} \rightarrow \mathbb{R}$ the solution to the following differential system

$$
\dot{\zeta}=-2 L \zeta-(1+\eta) \gamma\left(\zeta^{2}+1\right) \quad \zeta(0)=\theta^{-1},
$$

where $\theta \in(0,1)$ and $\eta>0$ is fixed. We denote $\widetilde{\mathcal{T}}(\theta, \eta, \gamma, L)$ the time it takes for $\zeta$ to decrease from $\theta^{-1}$ to $\theta$. This time $\widetilde{\mathcal{T}}(\theta, \eta, \gamma, L)$ is a continuous function of $\theta, \eta$ which 
is decreasing in $\theta, \eta$. On the other hand, we note that $\widetilde{\mathcal{T}}(\theta, \gamma, \eta, L) \rightarrow \mathcal{T}(\gamma, \eta, L)$ (where $\mathcal{T}(\gamma, \eta, L)$ is defined in (13)) as $\theta$ tends to 0 . As a consequence, since $T<$ $\mathcal{T}(\gamma, \eta, L)$, there exists $\theta$ such that $T<\tilde{\mathcal{T}}(\theta, \eta, \gamma, L)$. We fix the value of $\theta$.

Let $q:=(x, e, \tau)$. We define for all $q \in C \cup D$

$$
R(q):=V(x)+\max \left\{0, \gamma \zeta(\tau) W^{2}(e)\right\} .
$$

Let $q \in D$ and $G(q):=(x, 0,0)$. We obtain, in view of (7) and the fact that $W$ is positive semi-definite,

$$
\begin{aligned}
R(G(q)) & =V(x)+\max \left\{0, \gamma \zeta(0) W^{2}(0)\right\} \\
& =V(x) \leq R(q) .
\end{aligned}
$$

Let $q \in C$ and $(w, d, v) \in \mathbb{R}^{n_{w}+2 n_{d}}$ and suppose that $\zeta(\tau)<0$. As a consequence, $R(q)=V(x)$ and it holds that $\tau>T$. Indeed, $\zeta(\tau)$ is strictly decreasing in $\tau$, in view of (21), and $\zeta(T)>\zeta(\widetilde{\mathcal{T}})=\theta>0$ as $T<\widetilde{\mathcal{T}}$. Then $\zeta(\tau)<0$ implies that $\tau>T$. Hence, $\gamma^{2} W^{2}(e) \leq \delta(y)$ in view of (14) since $q \in C$. Consequently, in view of page 100 in [29], Lemma 1, Assumption 1 and (22)

$$
R^{\circ}(q ; F(q, w, d, v)) \leq-\alpha(|x|)+\mu(|(w, d, v)|),
$$

where $F(q):=(f(x, e, w, d), g(x, e, w, d, v), 1)$. Hence, in view of (9) and since $\alpha \in \mathcal{K}_{\infty}$, there exists $\rho_{1} \in \mathcal{K}_{\infty}$ such that

$$
\begin{aligned}
R^{\circ}(q ; F(q, w, d, v)) & \leq-\rho_{1}(V(x))+\mu(|(w, d, v)|) \\
& =-\rho_{1}(R(q))+\mu(|(w, d, v)|) .
\end{aligned}
$$

When $\zeta(\tau)>0$, we have

$$
R(q)=V(x)+\gamma \zeta(\tau) W^{2}(e) .
$$

As above, in view of page 100 in [29], Lemma 1, item (ii) of Assumption 1 and (21)

$$
\begin{aligned}
R^{\circ} & (q ; F(q, w, d, v))=\langle\nabla V(x), f(x, e, w, d)\rangle \\
& +\gamma \dot{\zeta}(\tau) W^{2}(e)+2 \gamma \zeta(\tau) W(e)\langle\nabla W(e), g(x, e, w, d, v)\rangle \\
\leq & -\alpha(|x|)-H^{2}(x)-\delta(y)+\gamma^{2} W^{2}(e)+\mu(|(w, d, v)|) \\
& +\gamma W^{2}(e)\left(-2 L \zeta-(1+\eta) \gamma\left(\zeta^{2}+1\right)\right) \\
& +2 \gamma \zeta(\tau) W(e)(L W(e)+H(x)+\sigma(|(w, d, v)|)) \\
\leq & -\alpha(|x|)-H^{2}(x)-\delta(y)-\eta \gamma^{2} W^{2}(e) \\
& +\mu(|(w, d, v)|)-(1+\eta) \gamma^{2} \zeta^{2}(\tau) W^{2}(e) \\
& +2 \gamma \zeta(\tau) W(e) H(x)+2 \gamma \zeta(\tau) W(e) \sigma(|(w, d, v)|) .
\end{aligned}
$$

Using several times the fact that $2 a b \leq \frac{1}{\kappa} a^{2}+\kappa b^{2}$ for any $a, b \geq 0$ and $\kappa>0$ and since $\delta(y) \geq 0$, we deduce that

$$
R^{\circ}(q ; F(q, w, d, v)) \leq-\alpha(|x|)-\eta \gamma^{2} W^{2}(e)+\chi(|(w, d, v)|),
$$

where $\chi:=\mu+\frac{1}{\eta} \sigma^{2}$. By using the same argument as in (25) and since $\zeta(\tau) \leq \theta^{-1}$ for all $\tau \geq 0$ in view of (21), we derive that

$$
\begin{aligned}
R^{\circ}(q ; F(q, w, d, v)) \leq & -\rho_{1}(V(x))-\rho_{2}\left(\gamma \zeta(\tau) W^{2}(e)\right) \\
& +\chi(|(w, d, v)|),
\end{aligned}
$$

where $\rho_{2}: s \mapsto \eta \gamma \theta s \in \mathcal{K}_{\infty}$. We deduce that there exists $\rho \in \mathcal{K}_{\infty}$ such that

$$
R^{\circ}(q ; F(q, w, d, v)) \leq-\rho(R(q))+\chi(|(w, d, v)|),
$$

where $\rho(s):=\min \left\{\rho_{1}\left(\frac{s}{2}\right), \rho_{2}\left(\frac{s}{2}\right)\right\}$ for $s \geq 0$.

When $\zeta(\tau)=0$, we obtain, in view of (25), (30) and Lemma 1

$$
R^{\circ}(q ; F(q, w, d, v)) \leq-\rho(R(q))+\chi(|(w, d, v)|) .
$$

Hence, (31) is satisfied in all cases.

Let $\phi:=\left(\phi_{x}, \phi_{e}, \phi_{\tau}\right)$ be a solution to (7), (14) with input $(w, d, v) \in \mathcal{L}_{\infty}$ and $\operatorname{dom}(w, d, v)=\operatorname{dom} \phi$. In view of (31) and page 99 in [29], it holds that, for some $\varepsilon \in(0,1)$,

$$
\begin{aligned}
\dot{R}(\phi(t, j)) \leq & R^{\circ}(\phi(t, j) ; F(\phi(t, j), w(t, j), d(t, j), v(t, j))) \\
\leq & -(1-\varepsilon) \rho(R(\phi(t, j)))-\varepsilon \rho(R(\phi(t, j))) \\
& +\chi(|(w(t, j), d(t, j), v(t, j))|)
\end{aligned}
$$

for all $j$ and for almost all $t \in I^{j}$ where $I^{j}=\{t:(t, j) \in$ dom $\phi\}$. As a consequence, using (23) and (32) and by following similar lines as in the end of the proof of Theorem 1 in [17], we deduce that for any $(t, j) \in \operatorname{dom} \phi$

$$
\begin{array}{r}
R(\phi(t, j)) \leq \max \{\bar{\beta}(R(\phi(0,0)), 0.5 t+0.5 T j), \\
\left.\rho^{-1}\left(\frac{1}{\varepsilon} \chi\left(\|(w, d, v)\|_{(t, j)}\right)\right)\right\} .
\end{array}
$$

On the other hand, in view of Assumption 1 and since $W$ is continuous (since it is locally Lipschitz) and positive semidefinite, there exists $\bar{\alpha}_{W} \in \mathcal{K}_{\infty}$ such that $W(e) \leq \bar{\alpha}_{W}(|e|)$ for all $e \in \mathbb{R}^{n_{e}}$ according to Lemma 4.3 in [30]. As a result, in view of Assumption 1, (21) and (22), it holds that, for all $q \in C \cup D$,

$$
\begin{aligned}
V(x) & \leq R(q) \leq V(x)+\frac{\gamma}{\theta} W^{2}(e) \\
\underline{\alpha}(|x|) & \leq R(q) \leq \bar{\alpha}(|x|)+\frac{\gamma}{\theta} \bar{\alpha}_{W}(|e|) \\
\underline{\alpha}(|x|) & \leq R(q) \leq \bar{\alpha}_{R}(|(x, e)|),
\end{aligned}
$$

where $\bar{\alpha}_{R}: s \mapsto \bar{\alpha}(s)+\frac{\gamma}{\theta} \bar{\alpha}_{W}(s) \in \mathcal{K}_{\infty}$. Hence, in view of (33) and (34), we deduce that

$$
\begin{aligned}
& \quad \underline{\alpha}\left(\left|\phi_{x}(t, j)\right|\right) \leq R(\phi(t, j)) \\
& \leq \max \left\{\bar{\beta}\left(\bar{\alpha}_{R}\left(\left|\left(\phi_{x}(0,0), \phi_{e}(0,0)\right)\right|\right), 0.5 t+0.5 T j\right),\right. \\
& \left.\rho^{-1}\left(\frac{1}{\varepsilon} \chi\left(\|(w, d, v)\|_{(t, j)}\right)\right)\right\} .
\end{aligned}
$$

Consequently,

$$
\begin{array}{r}
\left|\phi_{x}(t, j)\right| \leq \max \left\{\beta\left(\left|\left(\phi_{x}(0,0), \phi_{e}(0,0)\right)\right|, t+j\right),\right. \\
\left.\xi\left(||(w, d, v) \|_{(t, j)}\right)\right\},
\end{array}
$$

where $\beta:\left(s_{1}, s_{2}\right) \mapsto \underline{\alpha}^{-1}\left(\bar{\beta}\left(\bar{\alpha}_{R}\left(s_{1}\right), s_{2}\right)\right) \in \mathcal{K} \mathcal{L}, \xi(s):=$ $\underline{\alpha}^{-1}\left(\rho^{-1}\left(\frac{1}{\varepsilon} \chi(s)\right)\right)$ for $s \geq 0$. Thus, (15) holds. 